\title{
A concordant expression pattern of fatty acid synthase and membranous human epidermal growth factor receptor 2 exists in gastric cancer and is associated with a poor prognosis in gastric adenocarcinoma patients
}

\author{
HE LI, XUEFEI WANG, ZHAOQING TANG, FENGLIN LIU, WEIDONG CHEN, YONG FANG, \\ CONG WANG, KUNTANG SHEN, JING QIN, ZHENBIN SHEN*, YIHONG SUN* and XINYU QIN \\ Department of General Surgery, Zhongshan Hospital, Fudan University, Shanghai, P.R. China
}

Received August 15, 2014; Accepted April 14, 2015

DOI: $10.3892 / \mathrm{ol} .2015 .3609$

\begin{abstract}
Fatty acid synthase (FAS) and human epidermal growth factor receptor 2 (HER2) are overexpressed in gastric cancer (GC), and certain interactions have been found between FAS and HER2. A total of 94 patients were enrolled in the present study, each of whom underwent a D2 radical surgery in Zhongshan Hospital affiliated with Fudan University (Shanghai, China) between 2000 and 2005. The expression of FAS and HER2 was assessed by immunohistochemistry analysis of tissue microarrays generated from GC and non-tumor tissues. All data were analyzed by GraphPad Prism 5.0 to investigate the association between FAS and HER2 and to detect the potential association with prognosis. FAS $(\mathrm{P}<0.0001)$ and membranous HER2 (mHER2; $\mathrm{P}=0.0021$ ) were overexpressed in the GC tissues, and a bidirectional and strong correlation was demonstrated between FAS and mHER 2 in the tumor tissues. The expression of cytoplasmic HER2 (cHER2) was significantly lower in the GC tissues compared with the non-tumor tissues $(\mathrm{P}=0.0005)$, and cHER2 was expressed at a higher level in tumors that had better differentiation compared with poorly-differentiated tissues $(\mathrm{P}=0.0503)$. Patients with a concordant expression pattern of FAS and mHER2 showed a significantly poorer prognosis than the non-concordant group $(\mathrm{P}=0.0096$; hazards ratio, 3.2801; 95\% confidence interval, 1.5781-6.8176). GC tissues significantly overexpress FAS and mHER2 and the expression of these two markers is associated. Patients with a
\end{abstract}

Correspondence to: Professor Yihong Sun or Dr Zhenbin Shen, Department of General Surgery, Zhongshan Hospital, Fudan University, 180 Fenglin Road, Shanghai 200032, P.R. China

E-mail: sun.yihong@zs-hospital.sh.cn

E-mail: shen.zhenbin@zs-hospital.sh.cn

*Contributed equally

Key words: fatty acid synthase, human epidermal growth factor receptor 2, gastric cancer concordant expression of FAS and mHER2 are more likely to suffer a poor prognosis.

\section{Introduction}

Gastric cancer (GC) is a common disease and is the second leading cause of cancer-related mortality worldwide (1). Recently, significant developments have been made in the field of cancer-specific targeted therapy, and fatty acid synthase (FAS) and human epidermal growth factor receptor 2 (HER2) have emerged as possible markers of GC (2-4).

Fatty acids (FAs), which are components of the membrane and are essential in energy production, are absorbed from foods (exogenous pathway) or synthesized from intracellular substrates and enzymes (mainly through an endogenous pathway or de novo synthesis). FAS is a key biosynthetic enzyme involved in de novo synthesis, through which long chain FAs (LCFAs) can be produced with acetyl-CoA, malonyl-CoA and NADPH as substrates (5).

FAS is expressed in the liver, lipogenic tissues, proliferating fetal cells and hormone-sensitive cells, but is generally poorly expressed by non-tumor tissues (6-9). However, it has been reported that FAS is highly expressed in several cancers, including prostate, ovarian, breast, endometrial, thyroid, colorectal, bladder, gastric and lung cancers $(5,10)$. Moreover, this pattern of expression in transformed tissues cannot be modulated by physiological signals, as is occasionally the case in non-tumor tissues. Therefore, tumors can perpetually synthesize LCFAs to facilitate their proliferation and infiltration. At the same time, FAS is becoming increasingly significant in tumor diagnosis, prognosis and even treatment, particularly since a close association has been proposed between energy metabolism and tumor genesis $(5,11)$. Several in vitro studies have demonstrated the diagnostic value of FAS in cancer (12-14) or precancerous lesions (15). However, the role of FAS in gastric carcinogenesis has not been clearly identified.

FAS expression is modulated in multiple ways in cancer cells, one of which is through transcriptional regulation. Extracellular stimulants can ultimately activate FAS gene expression through the Ras/Raf/MAPK and PI3K/Akt 
pathways, but numerous other factors are also important, such as HIF-1 $\alpha$, mTOR and SPOT14 (16-18). In general, a complicated network of molecules is involved in FAS-related carcinogenesis, including HER2.

HER2 is a type of tyrosine kinase receptor that belongs to the erbB family. Similar to FAS, HER2 has been proven to be underexpressed in normal tissues, but in a number of tumors it is abnormally overexpressed and activated, including GC where patients with overexpression of HER 2 have a morbidity rate of $10-30 \%$ (19). HER2 can activate multiple downstream pathways, including the PI3K/Akt and Ras/Raf/MAPK pathways, which are the upstream signals of FAS. On the other hand, sufficient production of phospholipids for membrane microdomains will result in accommodation of receptor tyrosine kinases expressed on the membrane, including HER2 (20). Therefore, there appear to be certain significant correlations between FAS and HER2, which may synergistically modulate gastric carcinogenesis.

The roles that FAS has played in gastric carcinogenesis are under investigation. Overexpression of membranous HER2 (mHER2) in cancer tissues indicates a poor prognosis. Anti-HER2 therapy has been recommended in the treatment of HER2-positive GC patients (2), but the exact effect of this approach is yet to be determined. The experience gained from HER2-targeted therapy in breast cancer has shown that drug resistance inevitably interrupts the process of cancer treatment. Examinations of FAS and HER2 expression in breast $(21,22)$, ovarian (20) and oral (23) cancers has been performed in vivo. However, few studies have investigated FAS expression or its association with HER2 in GC. In the present study, FAS and HER 2 expression patterns were examined in 94 GC tissues and compared with adjacent non-tumor tissues. Finally, the expression of FAS and HER2, and their association with clinicopathological features and prognosis was examined in the GC patients.

\section{Materials and methods}

Ethics statement. The present study was approved by the Zhongshan Hospital Review Board (Shanghai, China), and all enrolled patients provided written informed consent to participate in the study.

Patients enrolled. A total of 94 patients with GC who underwent D2 surgery, performed by the same surgeon in Zhongshan Hospital between 2000 and 2005, were consecutively enrolled in this study. Prior to surgery, no therapy was administered to any of the patients. All patients had a complete clinicopathological history recorded, including age, gender, tumor size, histological grade, American Joint Committee on Cancer (AJCC) tumor stage, depth of invasion, lymph node metastasis and distant metastasis $(24,25)$. All patients presented with adenocarcinoma, and the median age of the patients at the time of diagnosis was 60 years (range, $30-80$ years). The histological grade of the tumor was evaluated under a microscope and was categorized based on the degree of tumor differentiation, tumor necrosis and mitotic count according to the criteria of Enzinger and Weiss $(26,27)$. Depth of invasion and lymph node metastasis were evaluated based on the National Comprehensive Cancer Network (NCCN) GC guideline (version 2011) (28). Follow-up time was calculated as the time of the initial surgery for the primary tumor until mortality or January 2013. The basic clinical information for all 94 patients is listed in Table I. Three of the patients presented positive for group no. 13 lymph node metastasis when enrolled and a D2 radical surgery was performed at that time according to the latest guidelines in 2011. These patients were labeled as exhibiting phase IV disease.

Tissue microarray (TMA) construction. From each patient, two cancerous and two non-tumor tissues $(5 \mathrm{~cm}$ away from the tumor edge) were obtained for TMA construction and immunohistochemical (IHC) staining. Non-tumor/healthy tissues were defined as the paired gastric tissues that were $5 \mathrm{~cm}$ away from the tumor edge. Tissue sections (diameter, $1.5 \mathrm{~mm}$; thickness, $4 \mu \mathrm{m}$ ) from archival, formalin-fixed, paraffin-embedded tissue specimens were mounted on poly-L-lysine-coated slides (Muto Chemicals, Tokyo, Japan). The sections were deparaffinized in xylene for $15 \mathrm{~min}$, rehydrated in different concentrations of ethanol and then heated at $95^{\circ} \mathrm{C}$ for $5 \mathrm{~min}$ in $10 \mathrm{mM}$ sodium citrate buffer ( $\mathrm{pH} \mathrm{6.0)} \mathrm{in}$ a microwave oven for antigen retrieval. Endogenous peroxidase was sequentially inactivated in $3 \% \mathrm{H}_{2} \mathrm{O}$ for 15 min at room temperature.

IHC staining of the TMAs. For FAS staining, the sections were blocked in 3\% normal donkey serum and subsequently incubated at $4^{\circ} \mathrm{C}$ overnight with monoclonal anti-FAS antibody (dilution, 1:50; \#3180; Cell Signaling Technology, Inc., Danvers, MA, USA). Finally, the sections were stained with horseradish peroxidase (HRP)-conjugated donkey anti-rabbit immunoglobulin $\mathrm{G}(\mathrm{H}+\mathrm{L})$ secondary antibody (Dako, Inc., Carpinteria, CA, USA).

For HER2 staining, the sections were first placed into a peroxidase-blocking reagent for $15 \mathrm{~min}$. The primary antibody (dilution, 1:10; \#2242; Cell Signaling Technology, Inc.) specific for HER2 was added and incubated at $4^{\circ} \mathrm{C}$ overnight. The sections were covered with Dako Envision+l HRP donkey anti-rabbit secondary antibody (Dako, Inc.) and incubated at room temperature for $30 \mathrm{~min}$. Signal detection was performed using a Dako signaling amplification system (product no. K346811). The TMA was counterstained with hematoxylin, then dehydrated and mounted for better tissue structure identification. Certain other routine reagents were provided by the Department of Pathology, Zhongshan Hospital (Shanghai, China).

IHC score of FAS and HER2. All the IHC-stained slides were interpreted by one pathologist blinded to the sample identities. IHC scoring of FAS and HER 2 was executed based on staining intensity and positivity. For each specimen, the staining intensity of FAS and cytoplasmic HER2 (cHER2) was scored as 0 for negative staining, 1 for weak intensity, 2 for moderate intensity and 3 for high intensity. The number of positive cells per section was categorized into three groups based on the percentage of positive cells: Group 1, <33\%; group 2, 33-67\%; and group 3, 68-100\%, which were scored as 1, 2 and 3 respectively (positivity score). This method of positive scoring was demonstrated by Vandhana et al in 2011 (29). Total scores according to the semiquantitative 
Table I. Complete clinical information of 94 gastric cancer patients.

\begin{tabular}{|c|c|}
\hline Characteristics & Value \\
\hline \multicolumn{2}{|l|}{ Gender, n (\%) } \\
\hline Male & $61(64.89)$ \\
\hline Female & $33(35.11)$ \\
\hline \multicolumn{2}{|l|}{ Age, years } \\
\hline Median & 60 \\
\hline Range & $30-80$ \\
\hline \multicolumn{2}{|c|}{ Histological type, n (\%) } \\
\hline Adenocacinoma & $94(100.00)$ \\
\hline Other & $0(0.00)$ \\
\hline \multicolumn{2}{|l|}{ Presentation, n (\%) } \\
\hline Initial & $94(100.00)$ \\
\hline Recurrent & $0(0.00)$ \\
\hline \multicolumn{2}{|l|}{ Size, cm } \\
\hline Median & 3 \\
\hline Range & $0.3-10$ \\
\hline \multicolumn{2}{|c|}{ Differentiation, n (\%) } \\
\hline I & $6(6.38)$ \\
\hline II & $34(36.17)$ \\
\hline III & $54(57.45)$ \\
\hline \multicolumn{2}{|l|}{ Metastasis, n (\%) } \\
\hline Negative & $91(96.81)$ \\
\hline Positive & $3(3.19)$ \\
\hline \multicolumn{2}{|l|}{ AJCC stage, n (\%) } \\
\hline I & $30(31.91)$ \\
\hline II & $18(19.15)$ \\
\hline III & $43(45.74)$ \\
\hline IV & $3(3.19)$ \\
\hline
\end{tabular}

AJCC, American Joint Committee on Cancer.

immunoreactivity scoring (IRS) method were obtained by multiplying the staining intensity by the positivity score, leading to a range from 0 to 9. Ultimate scores (US) of FAS [US(FAS)] and cHER2 [US(cHER2)] were defined as the average of each of the two total scores for one tissue (two sections). Next, US(FAS) and US(cHER2) was categorized into two grades using the area under curve method for further analysis as follows: Weak staining for US(FAS), $<6.75$; strong staining for US(FAS), $\geq 6.75$; weak staining for US(cHER2), <6; and strong staining for US(cHER2), $\geq 6$.

mHER2 IHC scoring was also performed based on intensity and positivity. The intensity of expression for each section was scored using four categories: $0+$, meaning that there was no membranous staining in any of the tumor cells; $1+$, meaning that there was membranous staining in $<10 \%$ of the tumor cells with any intensity or in $<30 \%$ of the tumor cells with weak intensity; $2+$, meaning that there was staining in 10-30\% of the tumor cells with moderate to strong intensity or staining in $30-50 \%$ of the tumor cells with weak to moderate intensity; and $3+$, meaning that there was staining in $>30 \%$ of the tumor cells with strong intensity or $>50 \%$ of the tumor cells with any intensity. The average of the two scores for the same tissue was defined as the US(mHER2) ranging from 0 to 3 . Tissues with a US(mHER2) score of $\leq 2$ were classified as overexpressed (positive) (30). The schematic of the IHC staining for FAS and HER2 is shown in Fig. 1A-H.

Statistical analysis. The data were analyzed with GraphPad Prism 5 (GraphPad Software, Inc., La Jolla, CA, USA) for Windows. The paired t-test was used to compare the FAS and HER2 expression levels in the cancer tissues with those in the non-tumor tissues. Contingency table analysis and $\chi^{2}$ tests were used to investigate the correlation between FAS and HER 2 protein expression and clinical parameters, and the Fisher's exact test was used when qualified. The correlation between FAS and HER2 was determined mainly by using the Mann-Whitney rank test or unpaired t-test. The survival rate was estimated using the Kaplan-Meier method. Any difference in survival curves was compared by Wilcoxon test and a hazard ratio was obtained. $\mathrm{P}<0.05$ was used to indicate a statistically significant difference.

\section{Results}

Overexpression of FAS in GC. The FAS expression pattern in the $94 \mathrm{GC}$ tissues and the adjacent non-tumor tissues was analyzed by TMA and IHC. FAS was expressed in the cell cytoplasm. A total of $54.3 \%$ (51/94) of the tumor tissues exhibited weak staining and 45.7\% (43/94) exhibited strong staining, whereas these values were $86.2 \%(81 / 94)$ and $13.8 \%(13 / 94)$ in the non-tumor tissues $\left(\mathrm{P}<0.0001 ; \chi^{2}\right.$ test), respectively. FAS was overexpressed in the GC tissues compared with the normal tissues $(5.633 \pm 0.510$ vs. $4.431 \pm 0.423 ; \mathrm{P}=0.0001$, Mann-Whitney test; Fig. 2A).

Overexpression of $m H E R 2$ in GC. HER2 was expressed not only in the cytoplasm, but also on the membrane. Using classification variables, the significance of mHER2 staining scores was determined by $\chi^{2}$ test, and overexpression of mHER2 was present in $21.3 \%$ (20/94) of the tumors and $5.3 \%$ $(5 / 94)$ of the non-tumor tissues $[\mathrm{P}=0.0021$; relative risk, 1.762; 95\% confidence interval (CI), 1.361-2.282; Fisher's exact test; Fig. 2B].

cHER2 is underexpressed in GC. cHER2 was found to be expressed in the GC and non-tumor tissues. In total, $44.7 \%$ of tumor tissues (42/94) exhibited strong staining (US(cHER2), $\geq 6$ ) for cHER2, while 63.8\% (60/94) of normal gastric tissues exhibited high expression levels of cHER2 $(\mathrm{P}=0.0126)$. Using the $\mathrm{t}$-test to determine significance, the tumor tissues were shown to underexpress cHER2 compared with the non-tumor tissues $(4.441 \pm 0.481$ vs. 5.662 \pm 0.465 ; $\mathrm{P}=0.0005$; Fig. 2C).

GC tissues exhibit a mutually strong correlation between FAS and mHER2. There is a potential interaction between FAS and mHER2 in the signaling pathway mentioned in the introduction, and the present study further combined these two molecules to analyze the correlation between them. mHER2 expression was significantly upregulated in the FAS-strong group compared with its control, and the expression of FAS in the mHER2-positive group was greater than its expression in the mHER2-negative group. These results documented a 

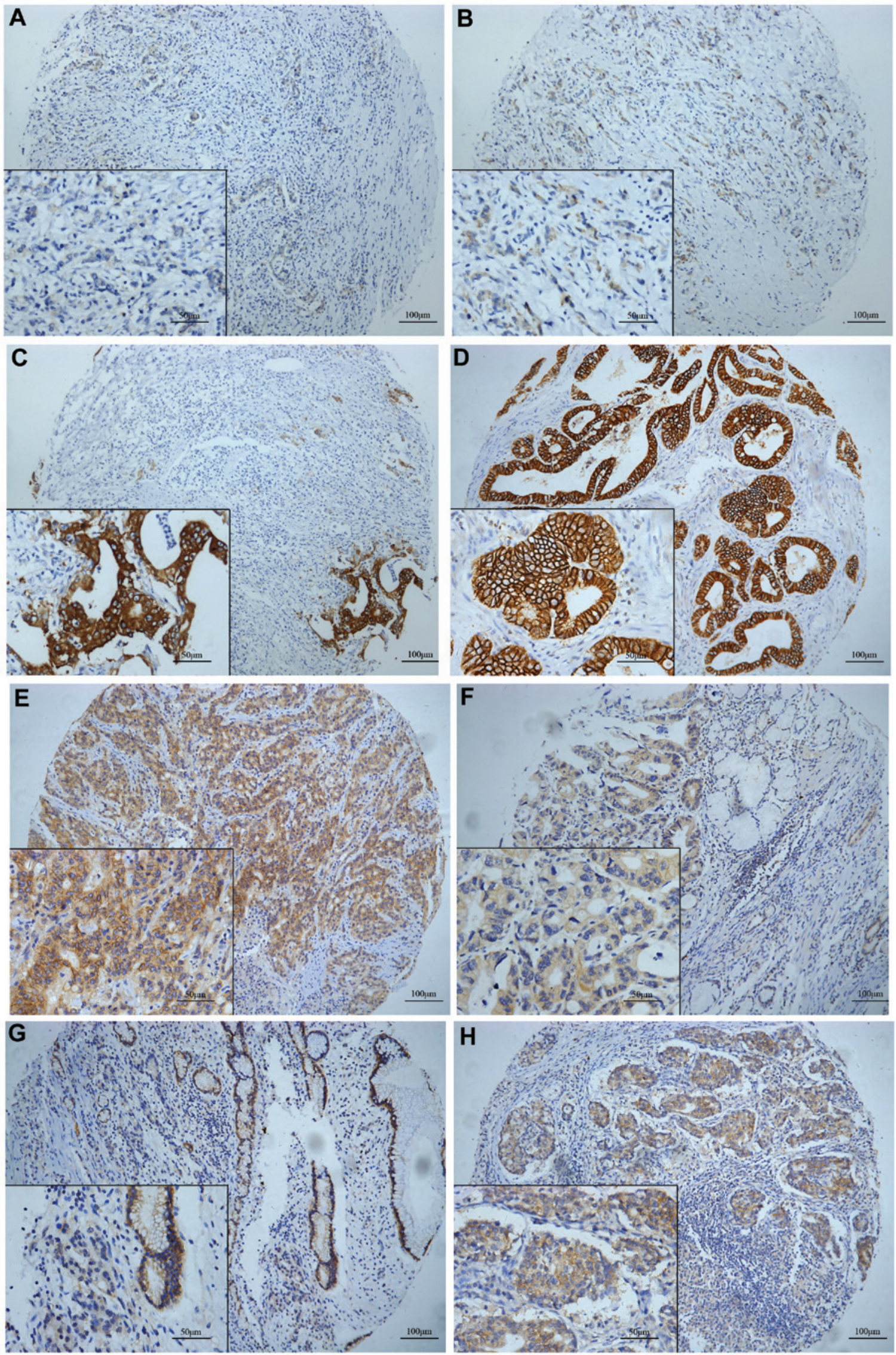

Figure 1. Fatty acid synthase (FAS) and human epidermal growth factor receptor 2 (HER2) expression in gastric cancer (GC) tissues. (A-C) Immunohistochemical staining of FAS in GC tissues. FAS was expressed in the cytoplasm according to different staining grades as follows: (A) Intensity, 1; positivity, 60\%; (B) intensity, 2; positivity, 70\%; and (C) intensity, 3; positivity, 90\%. The cytoplasm and membrane stained for HER2. (D) Cytoplasmic (c)HER2: Intensity, 0; memranous (m)HER2: Intensity, 3; positivity, 95\%; (E) cHER2: Intensity, 2; positivity, 90\%; mHER2: Intensity, 2; positivity, 60\%; (F) cHER2: Intensity, 2; positivity, 90\%; mHER2: Intensity, 1; positivity, 20\%; (G) cHER2: Intensity, 1; positivity, 60\%; mHER2: Intensity, 0; (H) cHER2: Intensity, 3; positivity, 80\%; mHER2: Intensity, 3; positivity, 20\%. Neither FAS or HER2 were expressed in the nucleus. (A-C, E, G and H) Poor differentiation. (D and F) Good or moderate differentiation. 
Table II. Correlation of FAS and mHER2 protein expression in gastric cancer with the clinicopathological characteristics of 94 patients.

\begin{tabular}{|c|c|c|c|c|c|c|c|c|c|c|c|c|c|}
\hline \multirow[b]{2}{*}{ Characteristic } & \multirow[b]{2}{*}{$\mathrm{n}$} & \multicolumn{3}{|c|}{ US(FAS) } & \multicolumn{3}{|c|}{ mHER2 } & \multicolumn{3}{|c|}{ cHER2 } & \multicolumn{3}{|c|}{ Combination of FAS and mHER2 } \\
\hline & & Weak & Strong & P-value & - & + & P-value & Weak & Strong & P-value & Concordant & Non-concordant & P-value \\
\hline Gender & & & & 0.0867 & & & 0.6078 & & & 0.5176 & & & 0.0439 \\
\hline Male & 61 & 29 & 32 & & 49 & 12 & & 32 & 29 & & 35 & 26 & \\
\hline Female & 33 & 22 & 11 & & 25 & 8 & & 20 & 13 & & 26 & 7 & \\
\hline Age, years & & & & 0.5348 & & & 0.0443 & & & 0.2130 & & & 0.8294 \\
\hline Median & & 58 & 62 & & 59 & 65 & & 57.5 & 63 & & 59 & 60 & \\
\hline Range & & $30-77$ & $43-80$ & & $30-77$ & $36-80$ & & $30-77$ & $40-80$ & & $30-80$ & $36-73$ & \\
\hline$<60$ & 48 & 28 & 20 & & 42 & 6 & & 30 & 18 & & 32 & 16 & \\
\hline$\geq 60$ & 46 & 23 & 23 & & 32 & 14 & & 22 & 24 & & 29 & 17 & \\
\hline Histological grade & & & & 0.1454 & & & 0.2158 & & & 0.0376 & & & 0.0484 \\
\hline $\mathrm{I}+\mathrm{II}$ & 40 & 18 & 22 & & 29 & 11 & & 17 & 23 & & 21 & 19 & \\
\hline III & 54 & 33 & 21 & & 45 & 9 & & 35 & 19 & & 40 & 14 & \\
\hline TNM stage & & & 0.4160 & & & 0.3183 & & & 0.5408 & & & 0.1995 & \\
\hline $0+1+2$ & 48 & 24 & 24 & & 40 & 8 & & 25 & 23 & & 28 & 20 & \\
\hline $3+4$ & 46 & 27 & 19 & & 34 & 12 & & 27 & 19 & & 33 & 13 & \\
\hline Depth of invasion & & & & 1.0000 & & & 0.1163 & & & 0.8324 & & & 0.1198 \\
\hline $\mathrm{T} 0+1+2$ & 35 & 19 & 16 & & 31 & 4 & & 20 & 15 & & 19 & 16 & \\
\hline $\mathrm{T} 3+4$ & 59 & 32 & 27 & & 43 & 16 & & 32 & 27 & & 42 & 17 & \\
\hline $\mathrm{LN}$ metastasis & & & & 0.4055 & & & 0.6126 & & & 0.5342 & & & 0.1892 \\
\hline N0 & 39 & 19 & 20 & & 32 & 7 & & 20 & 19 & & 22 & 17 & \\
\hline $\mathrm{N} 1+2+3$ & 55 & 32 & 23 & & 42 & 13 & & 32 & 23 & & 39 & 16 & \\
\hline Distant metastasis & & & & 1.0000 & & & 1.0000 & & & 1.0000 & & & 1.0000 \\
\hline M0 & 91 & 49 & 42 & & 71 & 20 & & 50 & 41 & & 59 & 32 & \\
\hline M1 & 3 & 2 & 1 & & 3 & 0 & & 2 & 1 & & 2 & 1 & \\
\hline Tumor size, $\mathrm{cm}$ & & & & 1.0000 & & & 0.3171 & & & 0.4073 & & & 1.0000 \\
\hline$<3$ & 38 & 21 & 17 & & 32 & 6 & & 19 & 19 & & 25 & 13 & \\
\hline$\geq 3$ & 56 & 30 & 26 & & 42 & 14 & & 33 & 23 & & 36 & 20 & \\
\hline Localization & & & & 0.9592 & & & 0.9689 & & & 0.1973 & & & 0.3279 \\
\hline $\mathrm{Up}$ & 10 & 5 & 5 & & 8 & 2 & & 3 & 7 & & 5 & 5 & \\
\hline Median & 44 & 24 & 20 & & 35 & 9 & & 27 & 17 & & 27 & 17 & \\
\hline Down & 40 & 22 & 18 & & 31 & 9 & & 22 & 18 & & 29 & 11 & \\
\hline
\end{tabular}

LN, lymph node; US, ultimate score; FAS, fatty acid synthase; mHER2, membranous human epidermal growth factor receptor 2; cHER2, cytoplasmic HER2; TNM, tumor-node-metastasis.

potent and bidirectional significant correlation between FAS and mHER 2 expression in the tumor tissues (a concordant expression pattern), but this pattern was not demonstrated in the non-tumor tissues (Fig. 3A and B).

A less differentiated state is associated with low cHER2 expression and is concordant with the expression of FAS and $m H E R 2$. The correlation between clinicopathological parameters, and the expression of FAS and HER2 was investigated. Clinical variables included age, gender, differentiation, AJCC stage, invasion depth, lymph node involvement, distant metastasis, tumor localization and tumor size. The results are listed in Table II. No significant correlations were detected between FAS and mHER2 expression. More significantly, a less differentiated state was associated with a concordant expression pattern [grade I+II vs. grade III; Fisher's exact test; P=0.0484; odds ratio (OR), 2.585; 95\% CI, 1.084-6.167] and reduced
cHER2 staining ( $\mathrm{P}=0.0376$; OR, 2.492; 95\% CI, 1.076-5.772). In addition, female patients appeared to suffer a much higher risk of a concordant expression pattern compared with male patients (Fisher's exact test; $\mathrm{P}=0.0439$; OR, 2.7595; 95\% CI, 1.039-7.330).

Concordant expression of FAS and HER2 indicates a poor prognosis in GC patients. Although it has been demonstrated that the in vitro overexpression of FAS and mHER2 commonly predicts a poor survival rate (20-23), the present data showed no significant overall survival difference between the groups classified by FAS ( $\mathrm{P}=0.4285$; Fig. 4A), mHER2 ( $\mathrm{P}=0.7094$; Fig. 4B) or cHER2 ( $\mathrm{P}=0.5507$; Fig. 4C). However, when combining mHER 2 and FAS together, and analyzing the data in the two groups as concordant or non-concordant, a prognostic difference was found between the groups. The survival curves of the patients are 

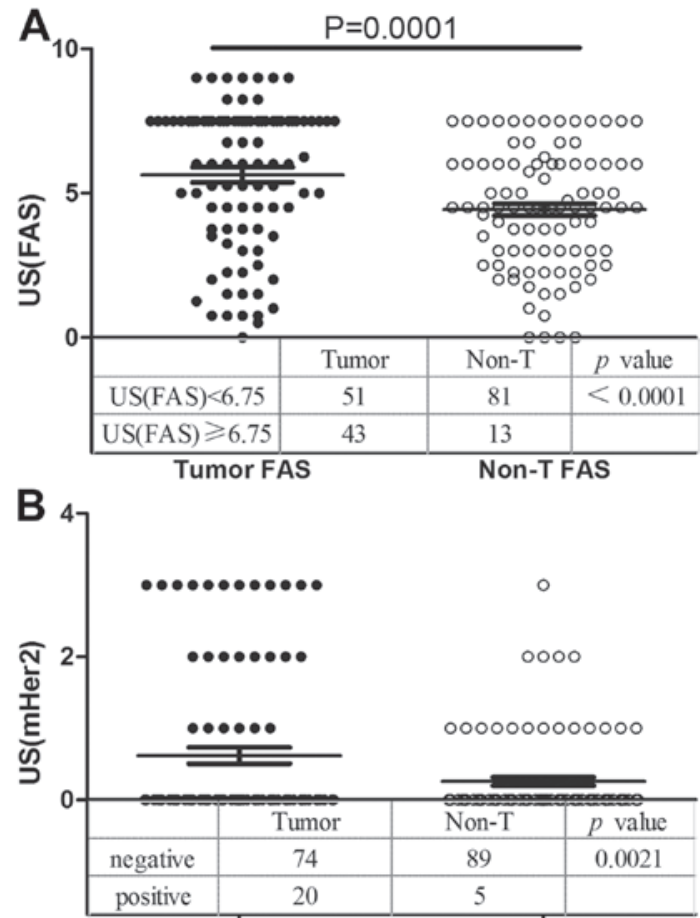

Tumor mHer2

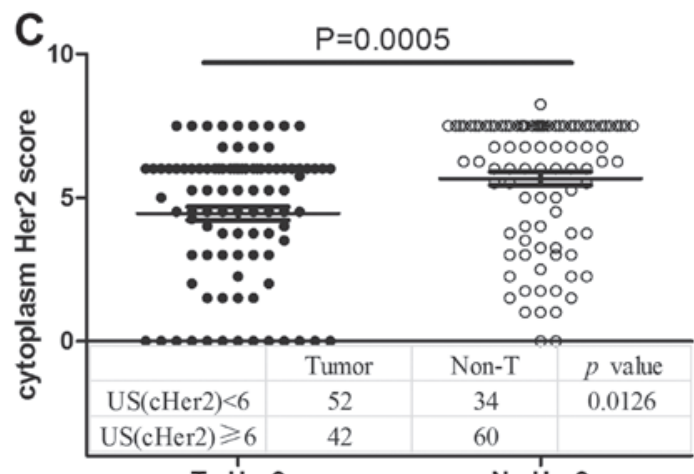

T cHer2

$\mathrm{N}$ cHer2
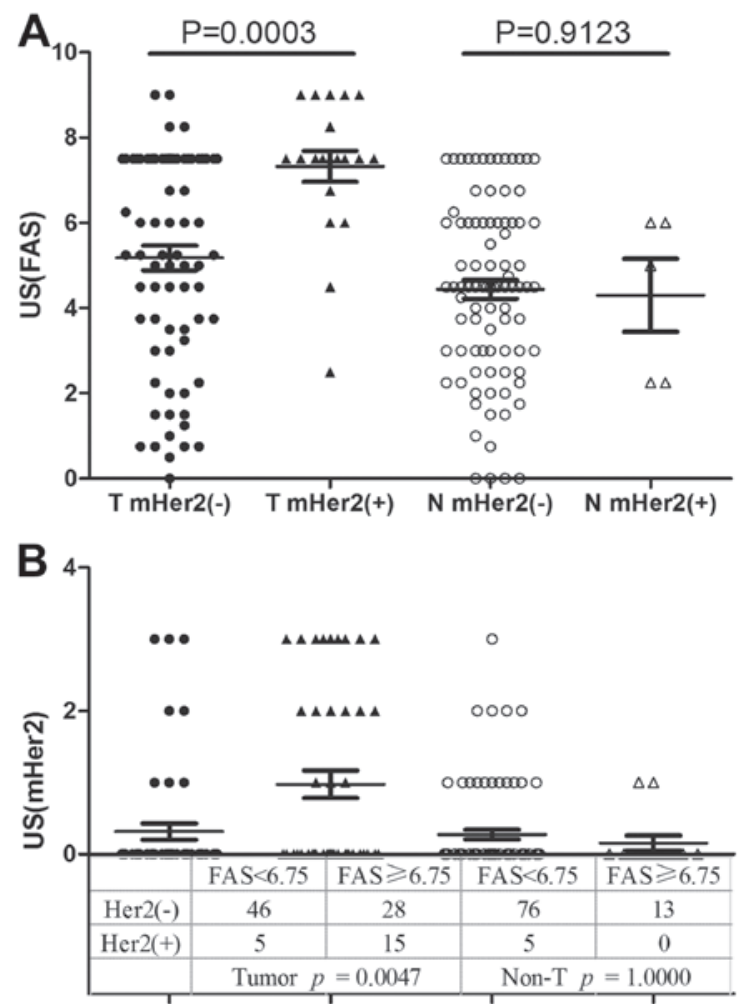

TFAS $<6.75 \quad$ TFAS $>6.75 \quad$ N FAS $<6.75 \quad$ N FAS $>6.75$

Figure 3. Co-expression pattern of FAS and mHER2 in gastric cancer tissues. (A) FAS expression shown according to mHER 2 status (5.176 \pm 0.578 vs. $7.325 \pm 0.761 ; \mathrm{P}=0.0003$ ). In the HER2-positive group, FAS was overexpressed compared with the HER2-negative group, but this correlation only existed in the tumor tissues and not the normal tissues. (B) mHER2 expression shown according to different FAS status in the tumor tissues ( $0.3137 \pm 0.2284$ vs. $0.9767 \pm 0.3886 ; \mathrm{P}=0.0047 ; \chi^{2}$ test). In non-tumor tissues, this phenomenon was not detected $(\mathrm{P}=1.0000)$. FAS, fatty acid synthase; mHER2, membranous human epidermal growth factor receptor 2; T, tumor tissue; N, non-tumor tissue; US, ultimate score.

Figure 2. Expression of FAS and HER2 in tumor and non-tumor (non-T) tissues. (A) Comparison of FAS expression between tumor and non-tumor tissues. The gastric cancer (GC) tissues overexpressed FAS (5.633 \pm 0.510 vs. $4.431 \pm 0.423 ; \mathrm{P}=0.0001$; Mann-Whitney rank test; or $\mathrm{P}<0.0001 ; \chi^{2}$ test). (B) Comparison of mHER2 staining status between tumor and non-tumor tissues. HER2 was significantly overexpressed in the GC tissues compared with the non-tumor tissues ( $\mathrm{P}=0.0027 ; \chi^{2}$ test). (C) Comparison of cHER 2 expression between tumor and non-tumor tissues. Unexpectedly, in contrast to mHER2, cHER2 was underexpressed in the GC tissues $(4.441 \pm 0.481$ vs. $5.662 \pm 0.465 ; \mathrm{P}=0.0005$; Mann-Whitney rank test; or $\mathrm{P}=0.0126 ; \chi^{2}$ test). FAS, fatty acid synthase; mHER2, membranous human epidermal growth factor receptor 2; cHER2, cytoplasmic HER2; US, ultimate score.

presented in Fig. 5A (Wilcoxon test; $\mathrm{P}=0.0096$; HR, 3.2801; 95\% CI, 1.5781-6.8176). When stratified by tumor differentiation, age and node metastasis, patients with concordant expression still showed a worse prognosis in differentiation grade III, elder and positive lymphatic metastasis patients (Fig. 5B-D).

\section{Discussion}

The treatment of GC has improved during the last few decades with regard to the surgical skills and tumor

targeted strategies, however, the general outcome for GC patients remains inadequate, and various studies have been conducted on gastric carcinogenesis and novel targeted molecules (2,31-33). FAS, a crucial synthesizer of LCFAs, is an enzyme that is involved in the synthesis of normal lipids and the development of cancer (5). FAS overexpression and increased activity represents one of the most recurrent phenotypic variations in cancer cells. A number of growth factors and their receptors, including HER2, have materialized as major contributors to the overexpression of FAS. However, the mechanisms ultimately responsible for tumor-associated FAS overexpression are not completely understood (5). In the present study, a potent and bidirectional correlation between FAS and mHER2 expression and the potential value for predicting patient outcome was primarily demonstrated in the GC patients. These novel findings may indicate an important role for these two combined molecules in gastric carcinogenesis and tumor invasiveness, and the potential benefits for future targeted therapy.

The present data showed that FAS and mHER2 were overexpressed in $45.7 \%$ (43/94) and $21.3 \%$ (20/94) of the GC tissues, respectively. The expression of these proteins in the cancer tissues was higher than their expression levels in the non-tumor tissues. 

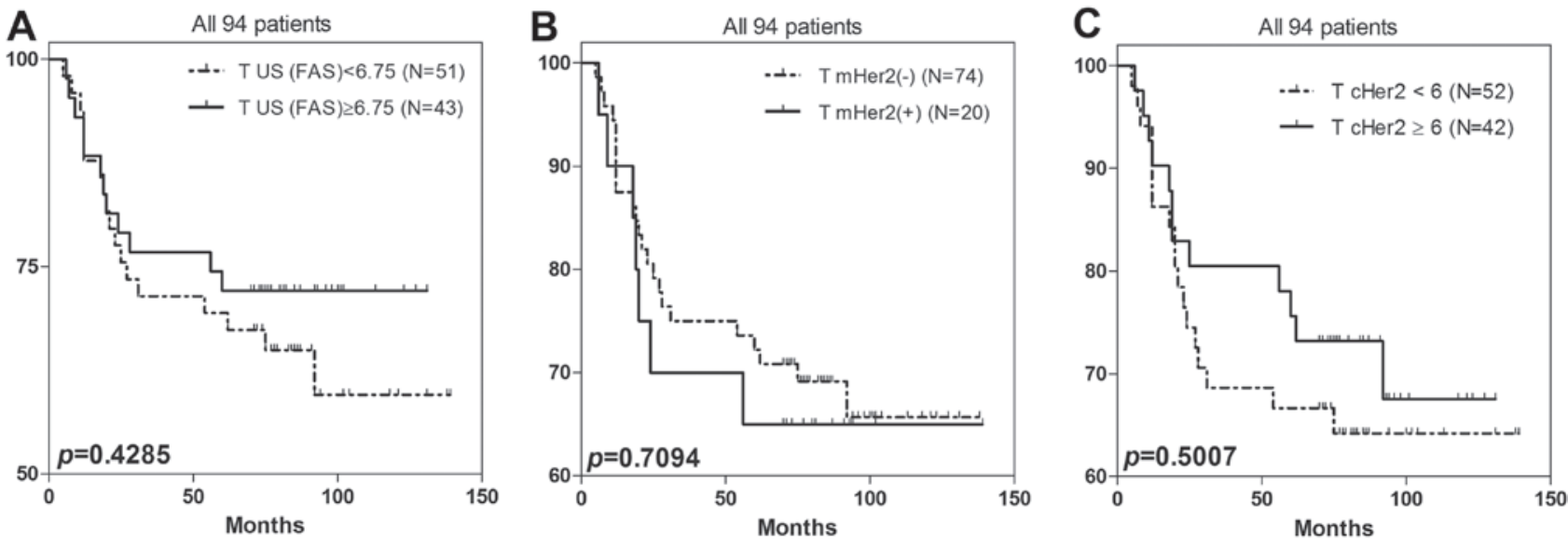

Figure 4. Survival analysis of all 94 GC patients. No correlation was detected between patient prognosis and the overexpression of FAS and HER2 when the data were analyzed separately. (A) Survival comparison by FAS in GC; P=0.4285; (B) survival comparison by mHER2 in GC; negative for a score of 0 and 1 , positive for a score of 2 and 3; $\mathrm{P}=0.7094$; (C) survival comparison by cHER2 in GC; weak for a score of $<6$, strong for a score of $\geq 6$; $\mathrm{P}=0.5007$. GC, gastric cancer; FAS, fatty acid synthase; mHER2, membranous human epidermal growth factor receptor 2; cHER2, cytoplasmic HER2; US, ultimate score.
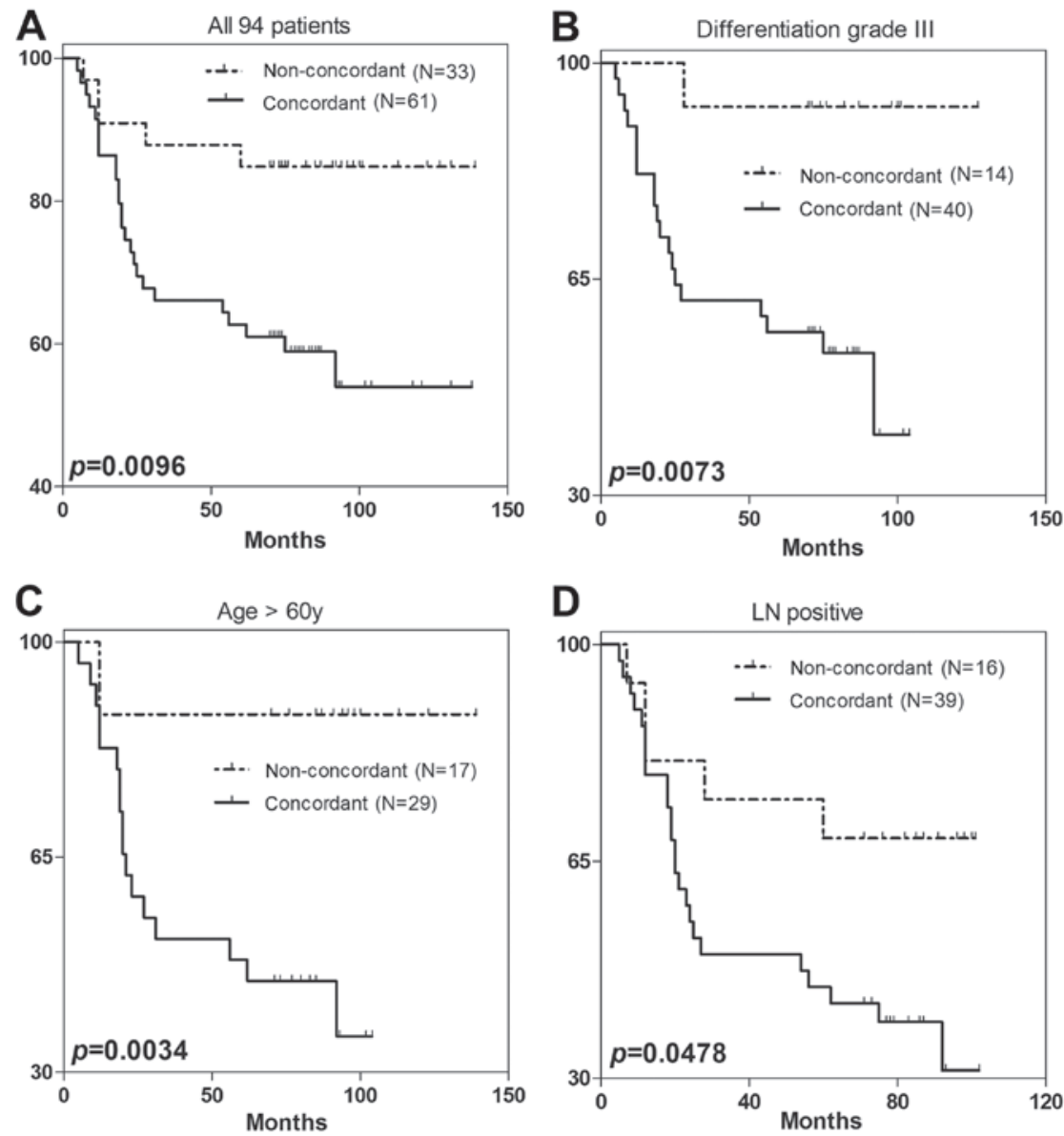

Figure 5. Prognostic analysis was conducted by combining FAS and mHER2 expression status. (A) There were significant differences in the survival outcomes in the two groups (Wilcoxon test; $\mathrm{P}=0.0096$; hazards ratio, 2.647; confidence interval, 1.267-5.532). The group with concordant expression of FAS and HER2 showed a worse outcome. When stratified by different (B) tumor differentiation grade, (C) patient age and (D) lymph node (LN) metastasis, the prognostic significance could still be obtained. FAS, fatty acid synthase; mHER2, membranous human epidermal growth factor receptor 2.

As shown in former studies, the positivity of FAS ranges from $50 \%$ to $>80 \%$ in various types of tumors $(15,34-38)$, apparently overexpressed in comparison with paired non-tumor tissues. In 2002, Kusakabe et al demonstrated the overexpression of FAS in GC tissues by IHC methods (39), and a following study was conducted to investigate the potential function of FAS in gastric carcinogenesis in vitro (40). HER2 as a membranous molecule has been reported to be overexpressed in various percentages of GC patients according to different studies (41-43). A study involving 1,414 GC 
patients showed that $17 \%$ of GC tissues would overexpress mHER2 (44). The present data was also consistent with these results. The mechanism of FAS and HER2 overexpression in cancer has been the subject of several studies; however, the total representation remains far from understood.

It has been hypothesized that there must be a potential correlation between FAS and mHER2 (45), with a number of studies confirming this fact, for example, in various tumors, including breast $(22,46,47)$, ovary $(20)$ or oral $(23,48)$ cancer, with mHER 2 and FAS upstream and downstream molecules of the PI3K and MAPK pathways. In normal human tissues, this type of correlation has not been found between FAS and mHER2. The present results showed that in GC tissues, the expression of FAS may be elevated along with mHER2 overexpression, and vice versa. This interaction appeared to serve as a positive-feedback pathway that can mutually regulate the expression of FAS and mHER2. This result showed that FAS and mHER2 were definitely correlated in GC, which was consistent with studies in other tumors (20-23).

The mechanisms involved in the mutual regulation between mHER2 and FAS have been mainly revealed. mHER2 activates the FAS gene promoter through the PI3K and MAPK signaling pathway, and finally elevates FAS expression. Moreover, mHER2 can directly activate FAS protein by its intracellular phosphorylation domain (22). Alternatively, HER2 gene expression and HER2 protein activity can be modulated through the concentration changes of acetyl-CoA and malonyl-CoA that are regulated by FAS $(5,49)$. In addition, as the key enzyme of de novo synthesis, FAS can increase the stability of mHER 2 by the formation of a domain known as a lipid raft, located on the membranes (46). These mechanisms ultimately construct a positive-feedback pathway between mHER2 and FAS.

The present study did not find any correlation between the clinical information and the expression of mHER 2 and FAS. Generally, mHER2 is considered to be highly correlated with intestinal GC (Laurén type) (50-54). However, information about Laurén type intestinal GC was not included in the present study. The reasons for this correlation between mHER2 and FAS, and the mechanisms behind it, remain to be elucidated. Other clinical parameters, including differentiation grade, tumor-node-metastasis stage and tumor size, were not confirmed to correlate with mHER 2 expression. FAS has been found to be highly expressed in well-differentiated GC tissues compared with poorly-differentiated GC tissues, and it also appears to function in the early stage of gastric carcinogenesis (39). This deduction was not statistically evident in the present study when the correlation between FAS expression and tumor differentiation was analyzed, but $55.0 \%(22 / 40)$ of grade I and II GC tissues demonstrated overexpression of FAS, and this ratio was $38.9 \%$ (21/54) in grade III tissues. So there may be a decreasing trend of FAS expression along with worsening tumor differentiation. In the generation of the majority of tumors, FA synthesis is a highly activated process to supply enough phospholipid and enzymes for the rapid proliferation of tumor cells. However, there is no standard FAS scoring system to evaluate its IHC staining level, which has shown variations and discrepancies among different studies $(3,4,39,40,55)$.

Currently, controversy remains with regard to the prognostic value of mHER2 in GC patients $(43,44,56,57)$, and former observational studies and the ToGA trial do not have a uniform conclusion to this issue (2,43,58-60). However, a number of recent studies have shown that elevated expression levels of mHER 2 are associated with tumor invasion and a poor prognosis $(56,61)$. However, the present study did not find that mHER2 exhibited prognostic value in GC patients, which may be due to several factors. GC only showed mHER2 positivity in $\sim 20 \%$ of the patients, but the sample capacity of the study was too limited to detect the potential and probable significance. Moreover, fluorescence in situ hybridization is commonly considered to be the gold standard in the evaluation of mHER2 expression (62), and other IHC methods may have a bias tendency. However, the consistency of these two methods is $\sim 93.5 \%$, as proposed by the ToGA trial (2). Moreover, the Herceptin standard was recommended by NCCN to evaluate the IHC staining of mHER2, and the present study used the criteria proposed by Chung et al in 2005 (30), thus it suggested that bias and deviations inevitably exist in spite of their good concordance. Finally, mHER2 is commonly overexpressed more simply in intestinal GC, but the Laurén type of GC in the present study was unknown, which may be a confounding factor in prognostic analysis, since a diffuse type definitely indicates an inferior prognosis. Therefore, after the patients were stratified by differentiation grade, it was found that in the poorly-differentiated groups, positive mHER2 expression significantly indicated a poor prognosis $(\mathrm{P}=0.0153)$, which may have resulted from a certain elimination of perplexing factors. On the other hand, this result showed that tumors with mHER 2 expression have a higher capability for invasion and metastasis, which is in disagreement with a former study (63). Therefore, the function and regulation of HER2-mediated pathways in gastric carcinogenesis are intricate and complex.

The value of FAS in predicting GC prognosis is not yet confirmed, although FAS has been considered to be correlated with the prognosis of various tumors, such as non-small cell lung carcinoma (37), melanoma (38) and soft-tissue sarcomas (64). It has been a more commonly accepted fact that FAS does not associate with the prognosis of GC patients. The present data did not find any significant correlation between FAS and patient survival, which is in agreement with a former study (39). In 2009, Dowling et al reported that FAS inhibitors could apparently induce the apoptosis of GC cells in vitro and depress tumor formation in mice, which to some extent reflect the potential roles of FAS in gastric carcinogenesis (40). This requires further investigation in more depth. However, notably, the present study found that the concordant expression group suffered a much worse prognosis compared with the non-concordant group, when FAS and mHER 2 were combined together in a survival analysis. The five-year overall survival rates were 62.7 and $84.8 \%$ in these two groups. Moreover, in the elder patients ( $>60$ years), the females and the patients with grade-III differentiation, concordant expression still acted as a predictor of a poor prognosis. It has been reported in vitro that prostate cells expressing FAS and androgen receptor (another activator of the PI3K-Akt pathway) can form invasive adenocarcinomas in immunodeficient mice, however, cells that expressed only FAS did not (65). Thus, we can hypothesize based on present data, that in non-tumor tissues, the positive-feedback pathway 
of mHER2-FAS is not activated, and no correlation was found between them. However, this pathway could be activated in a certain stage of gastric carcinogenesis and would promote gastric cell proliferation. Therefore, the patients that present with FAS/mHER 2 concordant expression may have a worse prognosis, due to the activation of the mHER2/FAS pathway.

However, not all GC patients presented with a pattern of concordant expression, and a large proportion of the patients showed non-concordant expression of FAS and mHER2, which may be a presentation of GC heterogenicity or result from other unknown mechanisms involved in the process of gastric carcinogenesis. Nevertheless, mHER2 and FAS are simultaneously modulated by various molecules in a complicated process, and more intensive and detailed cytological experiments are required to confirm or investigate this theory.

cHER 2 expressed in GC has seldom been investigated in depth. Unexpectedly, the present study found that cHER2 was significantly overexpressed in non-tumor tissues compared with tumor tissues. In addition, cancerous tissues with good differentiation (grade $\mathrm{I}+\mathrm{II}$ ) showed a higher percentage of cHER2 overexpression in comparison to poorly-differentiated cancerous tissues [57.5\% (23/40) vs. 35.2\% (19/54); $\mathrm{P}=0.0376$; Fisher's exact test]. It appears that the expression of cHER2 has a tendency to increase with GC progression. Therefore, cHER 2 may play completely different roles from mHER2, in normal and tumor tissues. With tumor progression, mHER2 increases and cHER2 decreases, and complicated mechanisms must be involved in this process.

Currently, the complications that arise from the use of HER2-targeted therapy in GC treatment are not fully understood, but the experience and information gained from HER2-targeted breast cancer therapy indicates that a large number of HER2-positive breast cancer patients are primarily resistant to anti-HER 2 drugs, and almost all of the patients will have drug-resistant tumors following HER2-targeted therapy (66-69). However, it has been proved in vitro that the combination of anti-FAS and anti-HER2 targeted therapy will decrease the resistance to HER2 inhibitors through various mechanisms $(21,22,70,71)$. Therefore, the present study preliminarily offers evidence for the feasibility of using combined detection of FAS and mHER 2 in GC patients, which appears most significant in mHER2-negative patients and mHER2-positive patients who are resistant to HER2-targeted therapy. Also, from the present results, we further surmise that this promising method will be of more use in the personalized treatment of GC patients who are $>60$ years old, female and who have tumors with poor differentiation. However, a great deal of research is required in order to achieve this goal, and more cytological and genetic studies are required.

In conclusion, FAS and mHER 2 are overexpressed in GC tissues, with a strong association with each other. The present study showed a concordant expression pattern of FAS and mHER2 in GC tissues, while there was no association between these two markers in healthy, adjacent non-tumor tissues. The two proteins are linked to the same signaling pathway (MAPK and PI3K/Akt), and their expression is associated with a poor prognosis for GC patients. cHER2 was also found to be underexpressed in GC tissues.

\section{Acknowledgements}

This study was supported by the Department of Pathology, Zhongshan Hospital, and GlaxoSmithKline (Philadelphia, PA, USA), who offered the use of their laboratories and equipment; the authors would like to express their appreciation for the assistance provided.

\section{References}

1. Devesa SS, Blot WJ and Fraumeni JF, Jr.: Changing patterns in the incidence of esophageal and gastric carcinoma in the United States. Cancer 83: 2049-2053, 1998.

2. Kuhajda FP: Fatty acid synthase and cancer: New application of an old pathway. Cancer Res 66: 5977-5980, 2006.

3. Notarnicola M, Tutino V, Calvani M, et al: Trastuzumab in combination with chemotherapy versus chemotherapy alone for treatment of HER2-positive advanced gastric or gastrooesophageal junction cancer (ToGA):A phase3, open-label, randomised controlled trial. Lancet 376: 687-697, 2010.

4. Ito T, Sako K, Maekawa H, et al: Elevated levels of serum fatty acid synthase in patients with gastric carcinoma. Oncol Lett 7: 616-620, 2014.

5. Menendez JA and Lupu R: Fatty acid synthase and the lipogenic phenotype in cancer pathogenesis. Nat Rev Cancer 7: 763-777, 2007.

6. Weiss L, Hoffmann GE, Schreiber R, et al: Fatty-acid biosynthesis in man, a pathway of minor importance. Purification, optimal assay conditions, and organ distribution of fatty-acid synthase. Biol Chem Hoppe Seyler 367: 905-912, 1986.

7. Wagle S, Bui A, Ballard Pl, et al:Hormonal regulation and cellular localization of fatty acid synthase in human fetal lung. Am J Physiol 227: L381-L390, 1999.

8. Kusakabe T, Maeda M, Hoshi N, et al: Fatty acid synthase is expressed mainly in adult hormone-sensitive cells or cells with high lipid metabolism and in proliferating fetal cells. J Histochem Cytochem 48: 613-622, 2000.

9. Pizer ES, Kurman RJ, Pasternack GR and Kuhjada FP: Expression of fatty acid synthase is closely linked to prolieration and stromal decidualization in cycling endometrium. Int J Gynecol Pathol 16: 45-51, 1997.

10. Kuhajada FP: Fatty acid synathase and cancer: New application of an old pathway. Cancer Res 66: 5977-5980, 2006.

11. Abramson HN: The lipogenesis pathway as a cancer target. J Med Chem 54: 5615-5638, 2011.

12. Tischler V, Fritzsche FR, Gerhardt J, et al: Comparison of the diagnostic value of fatty acid synthase (FASN) with alpha-methylacyl-CoA racemase (AMACR) as prostatic cancer tissue marker. Histopathology 56: 811-815, 2010.

13. Nguyen PL, Ma J, Chavarro JE, et al: Fatty acid synthase polymorphisms, tumor expression, body mass index, prostate cancer risk, and survival. J Clin Oncol 28: 3958-3964, 2010

14. Heo CK, Woo MK, Yu DY, et al: Identification of autoantibody against fatty acid synthase in hepatocellular carcinoma mouse model and its application to diagnosis of HCC. Int J Oncol 36: 1453-1459, 2010.

15. Ishimura N, Amano Y, Sanchez-Siles AA, et al: Fatty acid synthase expression in Barrett's esophagus: Implications for carcinogenesis. J Clin Gastroenterol 45: 665-672, 2011.

16. Furuta E, Pai SK, Zhan R, et al: Fatty acid synthase gene is up-regulated by hypoxia via activation of Akt and sterol regulatory element binding protein-1. Cancer Res 68: 1003-1011, 2008.

17. Calvisi DF, Wang C, Ho C, et al: Increased lipogenesis, induced by AKT-mTORC1-RPS6 signaling, promotes development of human hepatocellular carcinoma. Gastroenterology 140: 1071-1083, 2011.

18. Kim K, Kim HY, Cho HK, Kim KH and Cheong J: The SDF-1alpha/CXCR4 axis induces the expression of fatty acid synthase via sterol regulatory element-binding protein-1 activation in cancer cells. Carcinogenesis 31: 679-686, 2010.

19. Yan B, Yau EX, Bte Omar SS, et al: A study of HER2 gene amplification and protein expression in gastric cancer. $\mathrm{J}$ Clin Pathol 63: 839-842, 2010.

20. Grunt TW, Wagner R, Grusch M, et al: Interaction between fatty acid synthase- and ErbB-systems in ovarian cancer cells. Biochem Biophys Res Commun 385: 454-459, 2009. 
21. Oliveras G, Blancafort A, Urruticoechea A, et al: Novel anti-fatty acid synthase compounds with anti-cancer activity in HER2+ breast cancer. Ann N Y Acad Sci 1210: 86-92, 2010.

22. Jin Q, Yuan LX, Boulbes D, et al: Fatty acid synthase phosphorylation: A novel therapeutic target in HER2-overexpressing breast cancer cells. Breast Cancer Res 12: R96, 2010.

23. Silva SD, Perez DE, Alves FA, et al: ErbB2 and fatty acid synthase (FAS) expression in 102 squamous cell carcinomas of the tongue: Correlation with clinical outcomes. Oral Oncol 44: 484-490, 2008.

24. Rice TW, Blackstone EH and Rusch VW: 7th edition of the AJCC Cancer Staging Manual: Esophagus and Esophagogastric Junction. Ann Surg Oncol 17: 1721-1724, 2010.

25. Washington K: 7th edition of the AJCC Cancer Staging Manual: Stomach. Ann Surg Oncol 17: 3077-3079, 2010.

26. Weiss SW: Franz M. Enzinger: His life and work. Adv Anat Pathol 13: 109-113, 2006.

27. Kindblom LG: Lipomatous tumours - how we have reached our present views, what controversies remain and why we still face diagnostic problems: A tribute to Dr Franz Enzinger, Adv Anat Pathol 13: 279-285, 2006.

28. Ajana JA, Bathel JS, Bekaii-Saab T, et al: Gastric cancer. J Natl Compr Canc Netw 8: 378-409, 2010.

29. Vandhana S, Deepa PR, Jayanthi U, Biswas J and Krishnakumar S: Clinico-pathological correlations of fatty acid synthase expression in retinoblastoma: An Indian cohort study. Exp Mol Pathol 90: 29-37, 2011.

30. Chung KY, Shia J, Kemeny NE, et al: Cetuximab shows activity in colorectal cancer patients with tumors that do not express the epidermal growth factor receptor by immunohistochemistry. J Clin Oncol 23: 1803-1810, 2005.

31. Shah MA, Jhawer M, Ilson DH, et al: Phase II study of modified docetaxel, cisplatin, and fluororacil with bevacizymab in patients with metastatic gastroesophageal adenocarcinoma. J Clin Oncol 23: 1803-1810, 2005.

32. Shah MA, Power DG, Kindler HL, et al: A multicenter, phase II study of bortezomib (PS-341) in patients with unresectable or metastatic gastric and gastroesophageal junction adenomcarcinoma. Invest New Drugs 29: 1475-1481, 2011.

33. Doi T, Muro K, Boku N, et al: Multicenter phase II study of everolimus in patients with previously treated metastatic gastric cancer. J Clin Oncol 28: 1904-1910, 2010.

34. Gabrielson EW, Pinn ML, Testa JR and Kuhajda FP: Increased fatty acid synthase is a therapeutic target in mesothelioma. Clin Cancer Res 7: 153-157, 2001.

35. Orita H, Coulter J, Tully E, et al: High levels of fatty acid synthase expression in esophageal cancers represent a potential target for therapy. Cancer Biol Ther 10: 549-554, 2010.

36. Kearney KE, Pretlow TG and Pretlow TP: Increased expression of fatty acid synthase in human aberrant crypt foci: Possible target for colorectal cancer prevention. Int J Cancer 125: 249-252, 2009

37. Visca P, Sebastiani V, Botti C, et al: Fatty acid synthase (FAS) is a marker of increased risk of recurrence in lung carcinoma Anticancer Res 24: 4169-4173, 2004.

38. Kapur P, Rakheja D, Roy LC and Hoang MP: Fatty acid synthase expression in cutaneous melanocytic neoplasms. Mod Pathol 18: 1107-1112, 2005.

39. Kusakabe T, Nashimoto A, Honma K and Suzuki T: Fatty acid synthase is highly expressed in carcinoma, adenoma and in regenerative epithelium and intestinal metaplasia of the stomach. Histopathology 40: 71-79, 2002

40. Dowling S, Cox J and Cenedella RJ: Inhibition of fatty acid synthase by Orlistat accelerates gastric tumor cell apoptosis in culture and increases survival rates in gastric tumor bearing mice in vivo. Lipids 44: 489-498, 2009.

41. Allgayer H, Babic R, Gruetzner KU, Tarabichi A, Schildberg FW and Heiss MM: c-erbB-2 is of independent prognostic relevance in gastric cancer and is associated with the expression of tumor-associated protease systems. J Clin Oncol 18: 2201-2209, 2000.

42. Matsubara J, Yamada Y, Hirashima Y, et al: Impact of insulin-like growth factor type 1 receptor, epidermal growth factor receptor, and HER2 expressions on outcomes of patients with gastric cancer. Clin Cancer Res 14: 3022-3029, 2008.

43. Grabsch H, Sivakumar S, Gray S, Gabbert HE and Müller W: HER2 expression in gastric cancer: Rare, heterogeneous and of no prognostic value - conclusions from 924 cases of two independent series. Cell Oncol 32: 57-65, 2010.
44. Kim KC, Koh YW, Chang HM, et al: Evaluation of HER2 protein expression in gastric carcinomas: Comparative analysis of 1,414 cases of whole-tissue sections and 595 cases of tissue microarrays. Ann Surg Oncol 18: 2833-2840, 2011.

45. Kumar-Sinha C, Ignatoski KW, Lippman ME, Ethier SP and Chinnaiyan AM: Transcriptome analysis of HER2 reveals a molecular connection to fatty acid synthesis. Cancer Res 63: 132-139, 2003

46. Vazquez-Martin A, Fernandez-Real JM, Oliveras-Ferraros C, et al: Fatty acid synthase activity regulates HER2 extracellular domain shedding into the circulation of HER2-positive metastatic breast cancer patients. Int J Oncol 35: 1369-1376, 2009.

47. Vazquez-Martin A, Colomer R, Brunet J, Lupu R and Menendez JA: Overexpression of fatty acid synthase gene activates HER1/HER 2 tyrosine kinase receptors in human breast epithelial cells. Cell Prolif 41: 59-85, 2008.

48. Silva SD, Cunha IW, Rangel AL, et al: Differential expression of fatty acid synthase (FAS) and ErbB2 in nonmalignant and malignant oral keratinocytes. Virchows Arch 453: 57-67, 2008.

49. Menendez JA, Papadimitropoulou A, Vellon L and Lupu R: A genomic explanation connecting 'Mediterranean diet', olive oil and cancer: Oleic acid, the main monounsaturated fatty acid of olive oil, induces formation of inhibitory 'PEA3 transcription factor-PEA3 DNA binding site' complexes at the Her-2/neu (erbB-2) oncogene promoter in breast, ovarian and stomach cancer cells. Eur J Cancer 42: 2425-2432, 2006.

50. Lin JT, Wu MS, Shun CT, Lee WJ, Sheu JC and Wang TH: Occurrence of microsatellite instability in gastric carcinoma is associated with enhanced expression of erbB-2 oncoprotein Cancer Res 55: 1428-1430, 1995.

51. Wu MS, Shun CT, Wang HP, et al: Genetic alterations in gastric cancer: Relation to histological subtypes, tumor stage, and Helicobacter pylori infection. Gastroenterology 112: $1457-1465,1997$.

52. Polkowski W, van Sandick JW, Offerhaus GJ, et al: Prognostic value of Laurén classification and c-erbB-2 oncogene overexpression in adenocarcinoma of the esophagus and gastroesophageal junction. Ann Surg Oncol 6: 290-297, 1999.

53. Lemoine NR, Jain S, Silvestre F, et al: Amplification and overexpression of the EGF receptor and c-erbB-2 proto-oncogenes in human stomach cancer. Br J Cancer 64: 79-83, 1991.

54. Tanner M, Hollmén M, Junttila TT, et al: Amplification of HER-2 in gastric carcinoma: Association with Topoisomerase IIalpha gene amplification, intestinal type, poor prognosis and sensitivity to trastuzumab. Ann Oncol 16: 273-278, 2005.

55. Alli PM, Pinn ML, Jaffee EM, McFadden JM and Kuhajda FP: Fatty acid synthase inhibitors are chemopreventative for mammary cancer in neu-N transgenic mice. Oncogene 24: $39-46,2005$

56. Gravalos C and Jimeno A: HER2 in gastric cancer: A new prognostic factor and a novel therapeutic target. Ann Oncol 19: 1523-1529, 2008.

57. De Vita F, Giuliani F, Silvestris N, Catalano G, Ciardiello F and Orditura M: Human epidermal growth factor receptor 2 (HER2) in gastric cancer: A new therapeutic target. Cancer Treat Rev 36 (Suppl 3): S11-S15, 2010.

58. Lee KE, Lee HJ, Kim YH, et al: Prognostic significance of p53, nm23, PCNA and c-erbB-2 in gastric cancer. Jpn J Clin Oncol 33: 173-179, 2003

59. Yu GZ, Chen Y and Wang JJ: Overexpression of Grb2/HER2 signaling in Chinese gastric cancer: Their relationship with clinicopathological parameters and prognostic significance. J Cancer Res Clin Oncol 135: 1331-1339, 2009.

60. Park DI, Yun JW, Park JH, et al: HER-2/neu amplification is an independent prognostic factor in gastric cancer. Dig Dis Sci 51: 1371-1379, 2006.

61. Begnami MD, Fukuda E, Fregnani JH, et al: Prognostic implications of altered human epidermal growth factor receptors (HERs) in gastric carcinomas: HER 2 and HER 3 are predictors of poor outcome. J Clin Oncol 29: 3030-3036, 2011.

62. Pauletti G, Godolphin W, Press MF and Slamon DJ: Detection and quantitation of HER-2/neu gene amplification in human breast cancer archival material using fluorescence in situ hybridization. Oncogene 13: 63-72, 1996.

63. Hochwald SN, Kim S, Klimstra DS, Brennan MF and Karpeh MS: Analysis of 154 actual five-year survivors of gastric cancer. J Gastrointest Surg 4: 520-525, 2000.

64. Takahiro T, Shinichi K and Toshimitsu S: Expression of fatty acid synthase as a prognostic indicator in soft tissue sarcomas. Clin Cancer Res 9: 2204-2212, 2003 
65. Migita T, Ruiz S, Fornari A, et al: Fatty acid synthase: A metabolic enzyme and candidate oncogene in prostate cancer. J Natl Cancer Inst 101: 519-532, 2009.

66. Lan KH, Lu CH and Yu D: Mechanisms of trastuzumab resistance and their clinical implications. Ann N Y Acad Sci 1059: 70-75, 2005.

67. Nahta R, Yu D, Hung MC, Hortobagyi GN and Esteva FJ: Mechanisms of disease: Understanding resistance to HER2-targeted therapy in human breast cancer. Nat Clin Pract Oncol 3: 269-280, 2006.

68. Nahta R and Esteva FJ: Herceptin: Mechanisms of action and resistance. Cancer Lett 232: 123-138, 2006.

69. Hortobagyi GN: Trastuzumab in the treatment of breast cancer. N Engl J Med 353: 1734-1736, 2005.
70. Vazquez-Martin A, Colomer R, Brunet $\mathrm{J}$ and Menendez JA: Pharmacological blockade of fatty acid synthase (FASN) reverses acquired autoresistance to trastuzumab (Herceptin by transcriptionally inhibiting 'HER 2 super-expression' occurring in high-dose trastuzumab-conditioned SKBR3/Tzb100 breast cancer cells. Int J Oncol 31: 769-776, 2007.

71. Menendez JA, Vellon L and Lupu R: Targeting fatty acid synthase-driven lipid rafts: A novel strategy to overcome trastuzumab resistance in breast cancer cells. Med Hypotheses 64: 997-1001, 2005. 